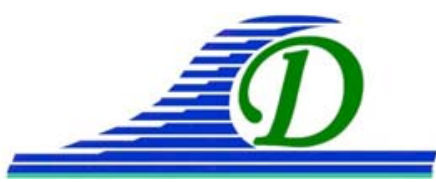

XIII ${ }^{\text {èmes }}$ Journées Nationales Génie Côtier - Génie Civil Dunkerque, 2-4 juillet 2014

DOI:10.5150/jngcgc.2014.013 @ Editions Paralia CFL

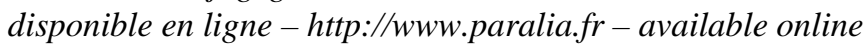

\title{
ANEMOC-2 Atlantique et Méditerranée : calibration et validation de deux nouvelles bases d'états de mer construites par simulations numériques rétrospectives sur 1979-2010
}

\author{
Amélie LAUGEL ${ }^{1}$, Anne-Laure TIBERI-WADIER ${ }^{2}$, \\ Michel BENOIT ${ }^{1,3}$, Giovanni MATTAROLO ${ }^{3}$
}

1. Laboratoire d'Hydraulique Saint-Venant, Université Paris-Est (ENPC, EDF R\&D, CEREMA), 6 quai Watier, BP 49, 78401 Chatou, France.

amelie.laugel@gmail.com,michel.benoit@saint-venant-lab.fr

2. CEREMA, 155 rue Pierre Bouguer, BP 5, 29280 Plouzané Cedex, France.

anne-laure.tiberi-wadier@cerema.fr

3. EDF R\&D Laboratoire National d'Hydraulique et Environnement (LNHE)

6, quai Watier, BP 49, 78401 Chatou, France.

giovanni.mattarolo@edf.fr

\section{Résumé :}

La caractérisation du climat de vagues, notamment grâce à la simulation numérique rétrospective ("hindcast") et la construction de bases d'états de mer, sont de premières importances pour de nombreuses activités maritimes, offshore et côtières. Par exemple, les bases rétrospectives d'états de mer ANEMOC (Atlas Numériques d'Etats de Mer Océanique et Côtier) sur les façades Atlantique, Manche, Mer du Nord (BENOIT \& LAFON, 2004 ; BENOIT et al., 2008) et Méditerranée (MORELLATO \& BENOIT, 2009) construites à l'aide du modèle spectral de $3^{\text {ème }}$ génération TOMAWAC (BENOIT et al., 1996) répondent à ces besoins. Au cours des trois dernières années, une version étendue et raffinée des deux atlas, appelée ANEMOC-2, a été construite, à la fois sur l’Océan Atlantique et la Mer Méditerranée. Ces deux nouvelles bases améliorent la connaissance du climat actuel de vagues aux abords des côtes françaises, par : (i) une extension de la période temporelle couverte (32 ans : 1979-2010), (ii) l'utilisation des champs de vent à $10 \mathrm{~m}$ de la réanalyse CFSR de résolution horaire et de résolution spatiale $0.312^{\circ}$; (iii) la construction de maillages plus fins à l'approche des côtes françaises (800 m à $1 \mathrm{~km}$ à la côte); (iv) une calibration des modèles à partir d'observations altimétriques de la base GlobWave et (v) une validation en milieu côtier et hauturier à l'aide des observations in situ des bouées (Bases Météo France, CANDHIS et RON).

Mots-clés : ANEMOC-2, Etats de mer, TOMAWAC, Hindcast, Océan Atlantique, Mer Méditerranée, Simulations spectrales de vagues, Calibration, Altimétrie. 


\section{Introduction}

Le Centre d'Études et d'Expertise sur les Risques, la Mobilité et l'Aménagement (CEREMA) et le Laboratoire National d'Hydraulique et Environnement (LNHE) d'EDF $R \& D$ collaborent depuis plusieurs années, notamment dans le cadre du Laboratoire d'Hydraulique Saint-Venant, à la construction de bases de données rétrospectives (hindcast) d'états de mer couvrant plusieurs décennies, sur les emprises de l'Océan Atlantique nord-est (BENOIT \& LAFON, 2004 ; BENOIT et al., 2008) et de la Mer Méditerranée (MORELLATO \& BENOIT, 2009). Pour le domaine Atlantique, deux maillages emboîtés, un premier appelé "océanique" et un second appelé "côtier", ont été les supports des simulations numériques opérées avec le code spectral de troisième génération TOMAWAC (BENOIT et al., 1996) à partir des forçages atmosphériques ERA-40 (UPPALA et al., 2005) sur la période 1979-2002. Le domaine Méditerranée a été simulé sur un unique maillage à l'aide de TOMAWAC et des forçages atmosphériques NCEP R2 (KALNAY et al., 1996) sur la période 1979-2008. Les deux bases ANEMOC "Atlas Numérique d'Etats de Mer Océanique et Côtier" sont disponibles depuis 2008 sur le site http://anemoc.cetmef.developpementdurable.gouv.fr/. Elles fournissent en différentes localisations de l'Atlantique nord-est et de la Méditerranée, (i) des séries temporelles des paramètres d'états de mer (hauteur significative $H_{\mathrm{m} 0}$, périodes énergétique $T_{\mathrm{E}}$, moyenne $T_{\mathrm{m} 02}$ et de pic $T_{\mathrm{p}}$, direction moyenne $\theta_{\mathrm{m}}$, étalement directionnel $\sigma$ et flux d'énergie linéique), (ii) des informations sur la climatologie moyenne annuelle et saisonnière des états de mer (histogrammes de distribution des paramètres synthétiques, corrélogrammes), et enfin (iii) des informations sur les valeurs extrêmes.

Dans l'objectif d'enrichir ces résultats et en complément des bases d'états de mer globales (ARDHUIN et al., 2011 ; REGUERO et al., 2012) ou régionales (GUEDES SOARES et al., 2002 ; DODET et al., 2010 ; CHARLES et al., 2012, BOUDIERE et al., 2013) qui n’offrent généralement pas de résolution suffisamment fine sur l'ensemble des côtes métropolitaines ou sur une période suffisamment longue, deux nouvelles versions des bases d'états de mer, nommées ANEMOC-2, ont été construites. Elles couvrent les emprises de l'Océan Atlantique où deux modèles "océanique" et "côtier" sont emboîtés, et de la Mer Méditerranée. Les améliorations suivantes ont été réalisées : (i) extension de la couverture temporelle, portée de 23 à 32 ans, de 1979 à 2010, (ii) amélioration des forçages atmosphériques en utilisant des réanalyses météorologiques plus récentes et de meilleure précision, (iii) réduction de la résolution le long des côtes françaises (de $800 \mathrm{~m}$ à $1 \mathrm{~km}$ ) et (iv) extension de l'emprise du modèle océanique. En complément, une attention particulière a été portée aux phases de calibration et validation des modèles. Ces dernières ont été respectivement menées à partir des observations altimétriques de la base GlobWave (QUEFFEULOU \& CROIZE-FILLON, 2009) et des observations in situ (Météo-France, CANDHIS http://candhis.cetmef.developpement-durable.gouv.fr/ et RON www.telemisura.it). 


\section{XIII ${ }^{\text {èmes }}$ Journées Nationales Génie Côtier - Génie Civil \\ Dunkerque, 2-4 juillet 2014}

L'article présente les modèles numériques utilisés pour la construction des deux atlas (section 2), la phase de calibration à l'aide des observations altimétriques (section 3) et la phase de validation à l'aide des mesures in situ de houlographes (section 4). Enfin, les conclusions et perspectives de ces travaux sont présentées en section 5.

\section{Modèle numérique}

2.1 TOMAWAC : code spectrale de simulation des états de mer.

Les simulations d'états de mer des bases ANEMOC-2 ont été réalisées à l'aide du modèle spectral de troisième génération TOMAWAC (BENOIT et al., 1996), faisant partie du système TELEMAC-MASCARET et développé au LNHE et au Laboratoire Saint-Venant. TOMAWAC résout l'équation de l'évolution d'action d'onde $N$ en espace et en temps (Equation 1) soumise aux interactions entre l'océan et l'atmosphère, la bathymétrie et le courant, dans un domaine océanique ou côtier discrétisé à l'aide d'un maillage non-structuré.

$$
\frac{\partial N}{\partial t}+\frac{\partial(\dot{x} N)}{\partial x}+\frac{\partial(\dot{y} N)}{\partial y}+\frac{\partial\left(\dot{k}_{x} N\right)}{\partial k_{x}}+\frac{\partial\left(\dot{k}_{y} N\right)}{\partial k_{y}}=Q\left(x, y, k_{x}, k_{y}, t\right)
$$

Le terme de droite $Q$ correspond aux processus physiques de génération des vagues par le vent, dissipation (moutonnement, frottement sur le fond, etc.) et transferts d'énergie au sein du spectre de vagues, pour lesquels différents modèles sont disponibles. Le tableau 1 résume les processus physiques et paramétrisations associées retenus lors des étapes de calibration des modèles océanique, côtier et Méditerranée d’ANEMOC-2.

Tableau 1. Paramétrisation des modèles océanique, côtier et Méditerranée d'ANEMOC-2 ; où X correspond aux processus physiques non pris en compte dans le modèle.

\begin{tabular}{|c|c|c|c|}
\hline & océanique & côtier & Méditerranée \\
\hline Génération des vagues par le vent & \multicolumn{3}{|c|}{ JANSSEN $(1989,1991)$} \\
\hline Dissipation par moutonnement & \multicolumn{2}{|c|}{ BAJ (BIDLOT et al., 2007) } & $\begin{array}{c}\text { KOMEN et al. (1984) } \\
\text { Coef. de dissipation : 3,2 } \\
\text { Coef. de pondération : 0,4 }\end{array}$ \\
\hline $\begin{array}{l}\text { Interactions entre quadruplets de } \\
\text { fréquences }\end{array}$ & \multicolumn{3}{|c|}{$\begin{array}{l}\text { Discrete Interaction Approximation (DIA) } \\
\text { (HASSELMANN \& HASSELMANN, 1985) }\end{array}$} \\
\hline $\begin{array}{l}\text { Dissipation par déferlement } \\
\text { bathymétrique }\end{array}$ & $X$ & $\begin{array}{c}\text { THORNTON \& } \\
\text { GUZA (1983) }\end{array}$ & $X$ \\
\hline $\begin{array}{l}\text { Dissipation par frottement sur le } \\
\text { fond }\end{array}$ & $X$ & $\begin{array}{l}\text { Campagne } \\
\text { JONSWAP }\end{array}$ & $X$ \\
\hline
\end{tabular}


Ainsi, en chaque point des maillages l'équation 1 est résolue avec une discrétisation de 32 fréquences de $0,034 \mathrm{~Hz}$ à $0,66 \mathrm{~Hz}$ (soit des périodes de $1,5 \mathrm{~s}$ à $29 \mathrm{~s}$ ) et 36 directions $\left(\Delta \theta=10^{\circ}\right)$ avec un pas de temps de $300 \mathrm{~s}$ pour le modèle océanique, $180 \mathrm{~s}$ pour le modèle côtier et $100 \mathrm{~s}$ pour le modèle Méditerranée. En chaque point du domaine, le spectre de variance est résolu, et les paramètres d'états de mer suivants sont conservés avec une résolution horaire : hauteur significative spectrale $H_{\mathrm{m} 0}$, périodes énergétique $T_{\mathrm{E}}$, moyenne $T_{\mathrm{m} 02}$ et de pic $T_{\mathrm{p}}$, direction moyenne $\theta_{\mathrm{m}}$, étalement directionnel $\sigma$ et flux d'énergie linéique des vagues.

\subsection{Maillages non-structurés}

Les figures 1 et 2 présentent respectivement les maillages Atlantique (océanique et côtier) et Méditerranée d'ANEMOC-2. Le modèle océanique (figure 1.a.) comporte 13426 nœuds et 22548 éléments et couvre l'Océan Atlantique de $63^{\circ} \mathrm{S}$ à $80^{\circ} \mathrm{N}$, des côtes américaines aux côtes européennes et africaines avec une résolution spatiale variant de $1,875^{\circ}$ au sud de l'Equateur à $0,6^{\circ}$ au nord de $20^{\circ} \mathrm{N}$ et à l'est de $40^{\circ} \mathrm{O}$ pour atteindre une résolution de $20 \mathrm{~km}$ le long des côtes européennes et de $10 \mathrm{~km}$ le long des côtes françaises (cf. figure 1.b.). Cette emprise permet de s'affranchir de la nécessité d'imposer des conditions aux limites du domaine, et de modéliser l'ensemble des états de mer se propageant vers les côtes françaises. Ainsi, le modèle océanique fournit les spectres de variance comme conditions limites au modèle côtier (cf . figure 1.c.), comprenant 16295 nœuds et 29018 éléments, et s'étendant de l’isobathe des $100 \mathrm{~m}$ jusqu'aux côtes françaises, où la résolution est de $1 \mathrm{~km}$, en incluant la Manche avec une résolution le long des côtes anglaises de 3 à 4 km.

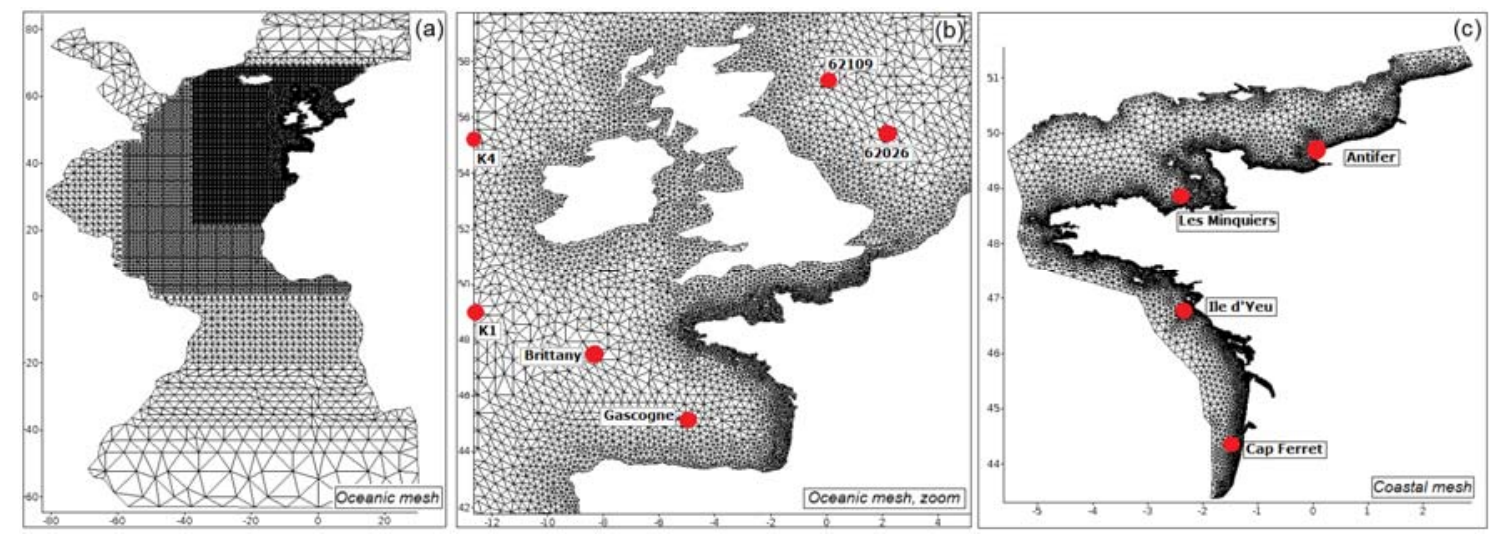

Figure 1. Maillages ANEMOC-2 des modèles océanique (a) avec un zoom sur les côtes européennes (b) et côtier (c) avec localisation de certaines bouées Météo-France et Candhis. 


\section{XIII ${ }^{\text {èmes }}$ Journées Nationales Génie Côtier - Génie Civil \\ Dunkerque, 2-4 juillet 2014}

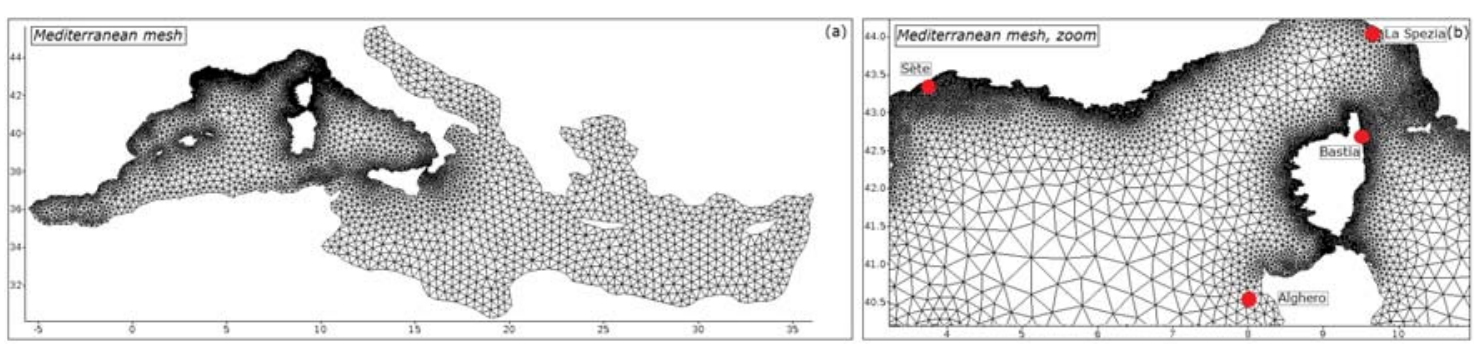

Figure 2. Maillage ANEMOC-2 du modèle Méditerranée (a) avec un zoom sur les côtes françaises (b) et localisation de certaines bouées Candhis et RON.

Le modèle Méditerranée (cf. figure 2.a.) comporte 15343 nœuds et 28159 éléments. Il couvre l'emprise complète de la Mer Méditerranée de $30^{\circ} \mathrm{N}$ à $46^{\circ} \mathrm{N}$ et de $6^{\circ} \mathrm{O}$ à $36^{\circ} \mathrm{E}$ avec une résolution variable de 40 à $50 \mathrm{~km}$ dans la partie Est et diminuant dans la partie Ouest du domaine pour atteindre $8 \mathrm{~km}$ le long des côtes italiennes et espagnoles et $800 \mathrm{~m}$ le long des côtes françaises (cf. figure 2.b). Les trois modèles ont été construits avec un niveau moyen de la mer, à partir des bases bathymétriques GEBCO (www.gebco.net) et Europe (issue du laboratoire LEGOS, Toulouse).

\subsection{Les champs de vent à $10 \mathrm{~m}$ de la réanalyse CFSR}

Les champs de vent à $10 \mathrm{~m}$ de la réanalyse Climate Forecast System Reanalysis (CFSR) (SAHA et al., 2010) ont été choisis comme forçages des bases ANEMOC-2. Ils ont été interpolés linéairement aux nœuds des trois maillages sur la période 1979-2010. Leurs résolutions fines à la fois en espace $\left(0,312^{\circ} \times 0,312^{\circ}\right)$ et en temps $(1 \mathrm{~h})$ ont motivé le choix de ce forçage atmosphérique.

\section{Calibration des bases ANEMOC-2}

\subsection{Les observations altimétriques GlobWave}

La calibration des trois modèles numériques (océanique, côtier et Méditerranée) d'ANEMOC-2 a été réalisée à partir des observations altimétriques de hauteurs significatives calibrées des vagues $H_{\mathrm{m} 0}$ de la base GlobWave (QUEFFEULOU \& CROIZE-FILLON, 2009) sur une période de 10 ans (2000-2009). Cette base regroupe les observations de huit satellites à partir de 1985, dont six sont disponibles sur la période 2000-2009 avec différentes couvertures temporelles et spatiales (répétitivité de 10, 17 ou 35 jours correspondant approximativement à une largeur au sol entre les traces de 240, 160 ou $80 \mathrm{~km}$ aux latitudes moyennes). Ainsi, la fréquence d'acquisition de $1 \mathrm{~Hz}$ ( $\sim \mathrm{km}$ aux latitudes moyennes) le long des traces altimétriques et le cumul de ces observations, recommandé par ALVES et YOUNG (2004) par exemple, offrent une couverture globale et quasi-continue d'observations de $H_{\mathrm{m} 0}$. A titre d'exemple sur la période 2000-2009, les satellites TOPEX/Poseidon, ERS-2, Geosat Follow On (GFO), Envisat, Jason-1et Jason-2 offrent un total de 7 à 15 millions d'observations annuelles 


\section{Thème 1 - Hydrodynamique côtière}

sur l'emprise de l'Océan Atlantique de $63^{\circ} \mathrm{S}$ à $63^{\circ} \mathrm{N}$. Ainsi, les six satellites sont utilisés pour la calibration du modèle océanique, calibration qui a ensuite été retenue pour le modèle côtier. Au contraire, seules les observations dont la couverture spatiale est la plus fine (Envisat et ERS-2) ont été conservées pour la calibration du modèle Méditerranée, avec l'objectif d’obtenir une répartition homogène des observations.

\subsection{Méthodologie en cinq étapes}

Pour définir la paramétrisation la plus précise des modèles ANEMOC-2, les observations GlobWave de $H_{\mathrm{m} 0}$ ont été comparées à différents résultats de simulations TOMAWAC à partir de la méthodologie suivante :

a) Extraction des observations altimétriques de $H_{\mathrm{m} 0}$ calibrées sur la zone d'intérêt et conservation des observations de qualité supérieure (indice 2 proposé par GlobWave, suppression des observations non-physiques, etc.) ;

b) Extraction des hauteurs de vagues $H_{\mathrm{m} 0}$ de TOMAWAC, et interpolation spatiotemporelle le long des traces altimétriques des satellites, afin d'avoir des données aux mêmes positions et instants ;

c) Moyenne des observations et résultats numériques toutes les $15 \mathrm{~s}$ pour le modèle océanique (pas de moyenne pour le modèle Méditerranée) ;

d) Répartition des observations et résultats numériques sur une grille de résolution $1^{\circ} \times 1^{\circ}$ sur l’Océan Atlantique et $0.5^{\circ} \times 0.5^{\circ}$ sur la Mer Méditerranée ;

e) Calculs des indices statistiques (Biais, RMSE, Scatter index, Symmetric Slope) sur les grilles et par zones d'intérêt: Golfe de Gascogne, Mer du Nord et Méditerranée Ouest (cf. cadres sur les figures 3 et 4).

Les paramètres testés pour les simulations avec TOMAWAC ont été de diverses natures : (i) différentes combinaisons des modèles représentant les processus physiques de génération, dissipation et transferts d'énergie, (ii) modification des coefficients de pondération de ces modèles, (iii) modification des champs de vent par correction quantile-quantile à partir d'observations de diffusiomètres, et enfin (iv) modification de la hauteur d'imposition des champs de vent.

\subsection{Résultats à l'issue de la calibration des modèles}

L'étape de calibration des modèles a abouti aux paramétrisations de TOMAWAC décrites dans le tableau 1, sans modification des champs de vent CFSR. Ainsi, les trois modèles partagent le modèle de génération des vagues par le vent selon JANSSEN (1989, 1991) et la représentation des interactions non-linéaires entre quadruplets de fréquences par l'approximation DIA de HASSELMANN \& HASSELMANN (1985). Néanmoins, la paramétrisation de la dissipation par moutonnement diffère entre les modèles. Pour les modèles océanique et côtier, la paramétrisation BAJ (BIDLOT et al., 2007), nouvellement implémentée dans TOMAWAC, a amélioré les processus de dissipation par moutonnement dans les cas de superposition des systèmes de houle de 


\section{XIII ${ }^{\text {èmes }}$ Journées Nationales Génie Côtier - Génie Civil \\ Dunkerque, 2-4 juillet 2014}

faibles fréquences et de la mer de vent, notamment dans la zone équatoriale. D’autre part, la paramétrisation du modèle Méditerranée modifie la formulation de la dissipation par moutonnement selon KOMEN et al. (1984) avec la diminution de la constante de dissipation à 3,2 et du coefficient de pondération à 0,4 dans l'objectif de diminuer légèrement l'intensité de ce terme de dissipation et d'obtenir des hauteurs significatives de vagues légèrement plus élevées. Enfin, les modèles de dissipation par déferlement bathymétrique selon THORNTON \& GUZA (1983) et par frottement sur le fond d'après la campagne JONSWAP (HASSELMANN et al., 1973) ont permis d'améliorer la propagation des vagues dans le domaine côtier (étape de calibration à partir d'observations in situ, non détaillée ici).

Les figures 3 et 4 illustrent la répartition spatiale du biais entre les simulations ANEMOC-2 et les observations altimétriques sur la période 2007-2009 (3 ans) pour le modèle océanique et sur la période 2003-2005 (3 ans) pour le modèle Méditerranée. Sur une large emprise des deux modèles, le biais entre les bases ANEMOC-2 et les observations évolue entre $+/-10 \mathrm{~cm}$, ce qui montre la bonne reproduction du climat moyen de hauteur significative par ANEMOC-2. En complément, le tableau 2 indique les résultats des indices statistiques (RMSE, Biais, Scatter Index et Symmetric Slope) moyennés sur les modèles complets et les zones principales d'intérêt que sont le Golfe de Gascogne, la Mer du Nord et la Méditerranée Ouest représentées par les cadres des figures 3 et 4 .

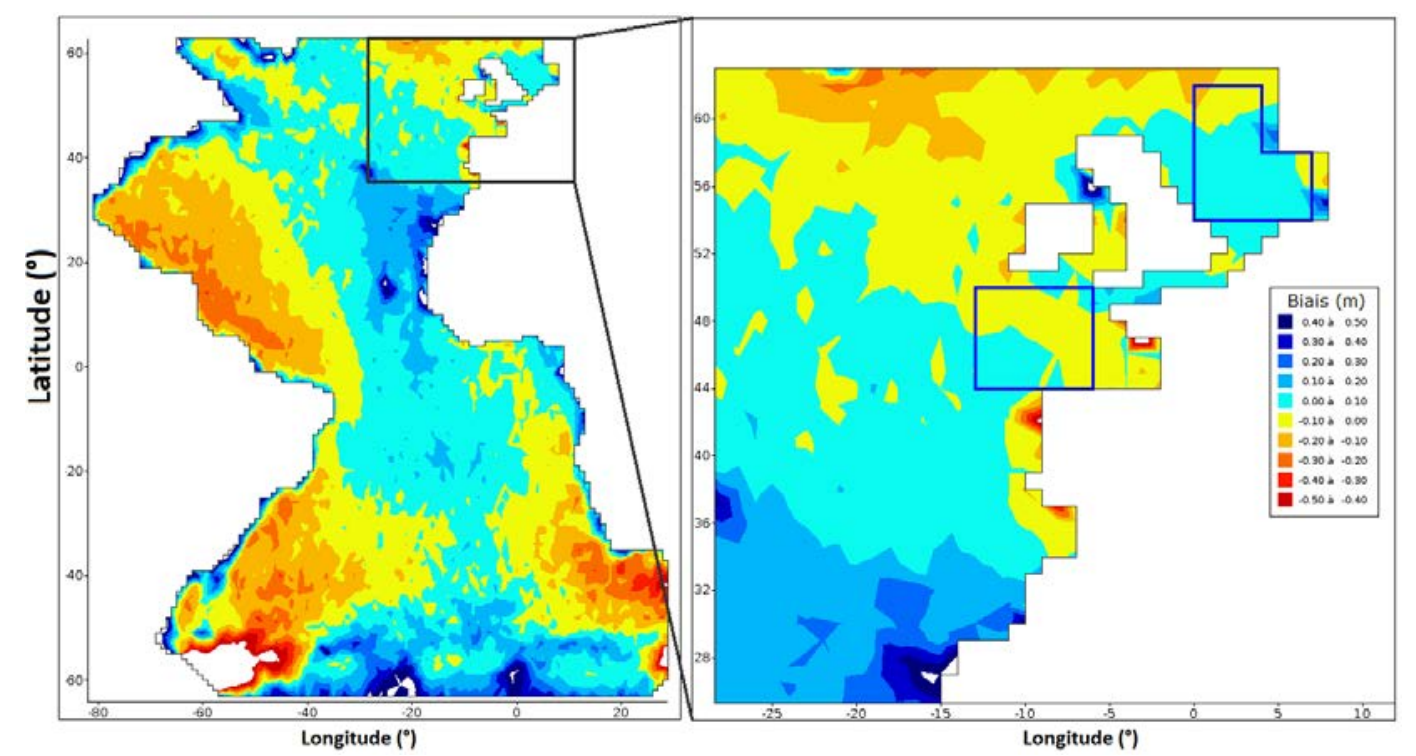

Figure 3. Répartition spatiale du biais de $H_{m 0}(m)$ entre le modèle ANEMOC-2 océanique et les observations GlobWave sur la période 2007-2009, en Atlantique, et avec un zoom sur les zones d'intérêt Golfe de Gascogne et Mer du Nord. 


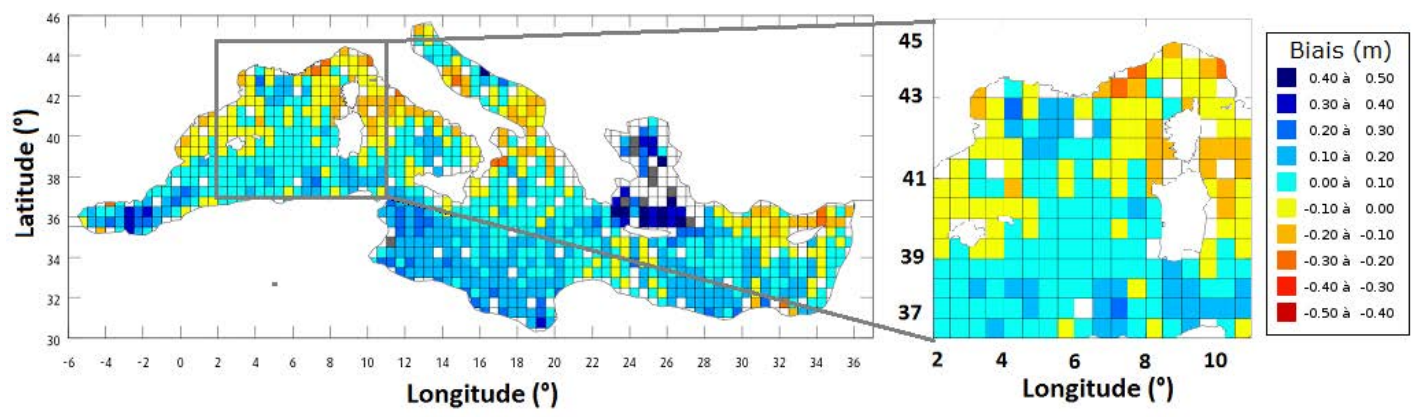

Figure 4. Répartition spatiale du biais de $H_{m 0}(m)$ entre le modèle ANEMOC-2 Méditerranée et les observations GlobWave sur la période 2003-2005, avec un zoom sur la zone d'intérêt "Méditerranée Ouest".

L'analyse des résultats de la comparaison montre que le modèle océanique a tendance à légèrement sous-estimer les observations altimétriques (biais compris entre -3 et $-6 \mathrm{~cm}$ ) alors que le modèle Méditerranée surestime légèrement les observations altimétriques (biais compris entre +3 et $+6 \mathrm{~cm}$ ). Notons que le RMSE du modèle océanique est plus important $(52 \mathrm{~cm})$ que pour l'ensemble des autres domaines (34 à $39 \mathrm{~cm}$ ), ce qui peut partiellement s'expliquer par la présence des archipels des Açores et du Cap Vert non modélisés dans ANEMOC-2, et les tailles de mailles plus grossières aux frontières sud et ouest du domaine.

Tableau 2. Comparaison des résultats ANEMOC-2 et observations GlobWave de $H_{m 0}$ sur la période 2000-2009 pour le modèle océanique et sur la période 2002-2008 pour le modèle Méditerranée.

\begin{tabular}{lllll}
\hline & RMSE $(\boldsymbol{m})$ & Biais $(\boldsymbol{m})$ & Scatter Index & Symmetric Slope \\
\hline Océan Atlantique & 0,52 & $-0,06$ & 0,17 & 0,95 \\
Golfe de Gascogne & 0,37 & $-0,03$ & 0,12 & 0,99 \\
Mer du Nord & 0,34 & $-0,05$ & 0,13 & 1,07 \\
Mer Méditerranée & 0,39 & 0,03 & 0,24 & 1,10 \\
Ouest Méditerranée & 0,38 & 0,06 & 0,26 & 1,13 \\
\hline
\end{tabular}

\section{Validation des modèles ANEMOC-2}

4.1 Motivation de cette étape de validation

Après le travail de calibration des modèles, présenté dans la partie précédente, nous avons souhaité procéder à une étape de validation indépendante des modèles. Pour cela nous avons utilisé des mesures différentes, issues de capteurs également différents (bouées houlographes). Cette étape de validation vise à gagner en confiance sur la précision et la qualité des résultats obtenus pour ANEMOC-2. 


\section{XIII ${ }^{\text {èmes }}$ Journées Nationales Génie Côtier - Génie Civil \\ Dunkerque, 2-4 juillet 2014}

\subsection{Les observations in situ Météo France, Candhis et RON}

Les observations in situ des bases de mesures de houlographes Météo-France, Candhis et RON, décorrélées des observations altimétriques, ont été utilisées pour valider la construction des modèles ANEMOC-2. Le tableau 3 résume les indices statistiques de Biais, RMSE et Scatter Index des hauteurs significatives $H_{\mathrm{m} 0}$ et période moyenne $T_{\mathrm{m} 02}$ entre les résultats d'ANEMOC-2 et les bouées localisées sur les figures 1.b., 1.c. et 2.b. Soulignons que les paramètres d'états de mer $\left(H_{m 0}, T_{m 02}\right.$, etc.) issus d'ANEMOC-2 sont calculés avec la contribution des hautes fréquences, y compris au-delà de l'intervalle de discrétisation du spectre (i.e. au-delà de $0,66 \mathrm{~Hz}$ ) alors que les résultats des observations in situ sont issus d'un spectre tronqué à une fréquence de coupure spécifique à chaque bouée (de l'ordre de $0,5 \mathrm{~Hz}$ à $0,625 \mathrm{~Hz}$ ). Une comparaison des paramètres réduits calculés sur un intervalle de fréquences identique pour les résultats de simulations et les observations, améliorerait très certainement la comparaison, notamment pour $T_{m 02}$.

L'analyse des valeurs du tableau 3 montre la capacité des modèles ANEMOC-2 à reproduire les observations in situ de $H_{\mathrm{m} 0}$ avec un faible biais négatif pour les modèles océanique et Méditerranée ( $\sim-10 \mathrm{~cm}$ pour la plupart des observations) et un faible biais positif pour le modèle côtier ( $\sim 16$ à $+19 \mathrm{~cm})$. A l'exception des bouées Île d'Yeu et Brittany, les résultats montrent une sous-estimation de $T_{\mathrm{m} 02}$ de la part d'ANEMOC-2 de l'ordre de $-1 \mathrm{~s}$ au maximum en milieu hauturier et proche de $-0,7 \mathrm{~s}$ en milieu côtier.

Tableau 3. Validation des modèles ANEMOC-2 $\left(\mathrm{H}_{m 0} ; \mathrm{T}_{m 02}\right)$ par rapport aux observations in situ de bouées houlographes (Biais, RMSE, Scatter Index).

\begin{tabular}{|c|c|c|c|c|c|c|c|c|c|}
\hline & \multirow[t]{3}{*}{ Bouée } & \multirow[t]{3}{*}{ Durée } & \multirow{3}{*}{$\begin{array}{l}\text { Bathymétrie } \\
\text { (m) }\end{array}$} & \multirow{2}{*}{$\begin{array}{l}\text { Biais } \\
\boldsymbol{H}_{m 0} \\
\end{array}$} & \multirow{3}{*}{$\begin{array}{l}T_{m 02} \\
(s)\end{array}$} & \multicolumn{2}{|c|}{ RMSE } & \multicolumn{2}{|c|}{ Scatter Index } \\
\hline & & & & & & $\boldsymbol{H}_{m 0}$ & $T_{m 02}$ & $H_{m 0}$ & $T_{m 02}$ \\
\hline & & & & (cm) & & (cm) & (s) & & \\
\hline \multirow{6}{*}{ : } & Gascogne & 1998-2009 & 4500 & -10 & $-0,90$ & 44 & 1,28 & 0,18 & 0,18 \\
\hline & Brittany & 1996-2009 & 2100 & -11 & 0,89 & 47 & 1,19 & 0,17 & 0,16 \\
\hline & 62026 & $1996-2001$ & 35 & -10 & $-0,88$ & 48 & 1,32 & 0,26 & 0,24 \\
\hline & 62109 & $1995-2001$ & 80 & 6 & $-0,83$ & 45 & 1,49 & 0,32 & 0,19 \\
\hline & K4 & 1999-2001 & 2900 & -2 & $-0,62$ & 60 & 1,12 & 0,18 & 0,15 \\
\hline & K1 & 1996-2001 & 1900 & -2 & $-0,60$ & 52 & 1,07 & 0,17 & 0,15 \\
\hline \multirow{5}{*}{ 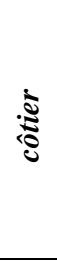 } & Cap Ferret & 2001-2002 & 54 & -11 & $-0,73$ & 33 & 1,13 & 0,19 & 0,17 \\
\hline & & 2007-2009 & & & & & & & \\
\hline & Île d’Yeu & 2005-2009 & 14 & 0,19 & 0,58 & 33 & 1,03 & 0,29 & 0,29 \\
\hline & Antifer & 1996-2009 & 17 & 15 & $-0,62$ & 29 & 1 & 0,46 & 0,23 \\
\hline & Les Minquiers & 1992-2009 & 38 & 16 & $-0,69$ & 31 & 1,19 & 0,27 & 0,22 \\
\hline \multirow{4}{*}{ 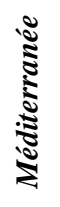 } & Sète & 2006-2009 & 32 & -9 & $-0,6$ & 5 & 0,6 & 0,30 & 0,27 \\
\hline & Bastia & 2006-2008 & 17 & -8 & $-1,0$ & 3 & 0,4 & 0,37 & 0,30 \\
\hline & La Spezia & 1998-2006 & 90 & -13 & $-0,5$ & 6 & 1,5 & 0,28 & 0,30 \\
\hline & Alghero & 1998-2007 & 95 & -3 & $-0,8$ & 8 & 2 & 0,20 & 0,30 \\
\hline
\end{tabular}




\section{Conclusions et perspectives}

Deux nouvelles bases d'états de mer ANEMOC-2 ont été construites sur la période 1979-2010 (32 ans) à l'aide du code spectral TOMAWAC et des champs de vent de la réanalyse CFSR sur les emprises de l’Océan Atlantique et de la Mer Méditerranée. Calibrées à l'aide des observations altimétriques sur la période 2000-2009 et validées à l'aide des observations in situ sur l'ensemble de leurs périodes disponibles, les bases ANEMOC-2 mettent à disposition différents paramètres d'états de mer $\left(H_{\mathrm{m} 0}, T_{\mathrm{E}}, T_{\mathrm{m} 02}\right.$, $\left.T_{\mathrm{p}}, \theta_{\mathrm{m}}, \ldots\right)$ avec une résolution temporelle et spatiale fine $(1 \mathrm{~h}, 20 \mathrm{~km}$ le long des côtes européennes et 0,8 à $1 \mathrm{~km}$ le long des côtes françaises).

Différents axes de recherche sont en cours afin d'améliorer encore la qualité des bases ANEMOC-2. Citons notamment la prise en compte des interactions vagues-courant par le couplage entre le code de courantologie TELEMAC-2D (pour modéliser la marée et les surcotes-décotes météorologiques) et TOMAWAC, qui améliorera la modélisation des états de mer dans les zones de forts marnages (en Manche notamment). En complément, la décomposition spectrale des états de mer permettra de différentier les systèmes de houle et de mer de vent, et la mise à jour de la base à l'aide des forçages atmosphériques CFSRv2 augmentera la couverture temporelle des atlas pour les années 2011 et suivantes.

\section{Remerciements :}

Les auteurs remercient la NOAA pour la mise à disposition de la réanalyse CFSR utilisée dans ce travail. De même, les instituts IFREMER, Météo-France, CEREMA, ISPRA (RON), LEGOS et GEBCO sont remerciés pour l'exploitation respective des observations altimétriques, diffusiomètres, observations in situ et bases bathymétriques.

\section{Références bibliographiques}

ALVES J., YOUNG I. (2004). On estimating extreme wave heights using combined Geosat, Topex/Poseidon and ERS-1 altimeter data. Applied Ocean Research, Vol. 25, pp 167-186. http://dx.doi.org/10.1016/j.apor.2004.01.002

ARDHUIN F., HANAFIN J., QUILFEN Y., CHAPRON B., QUEFFEULOU P., OBREBSKI M., SIENKIEWICZ J. VANDEMARK D. (2011). Calibration of the IOWAGA global wave hindcast (1991-2011) using ECMWF and CFSR winds. Proc. $12^{\text {th }}$ International Workshop of Wave Hindcasting and Forecating, Hawaii, (USA).

BENOIT M., LAFON F. (2004). A nearshore wave atlas along the coasts of France based on the numerical modelling of wave climate over 25 years. Proc. 29th Int. Conf. on Coastal Eng. (ICCE’2004). Lisbonne (Portugal). pp 714-726.

BENOIT M., MARCOS F., BECQ F. (1996). Development of a third generation shallow-water wave model with unstructured spatial meshing. Proc. 25th Int. Conf. on Coastal Engineering, Orlando, FL (USA), ASCE, pp 465-478. 


\section{XIII ${ }^{\text {èmes }}$ Journées Nationales Génie Côtier - Génie Civil \\ Dunkerque, 2-4 juillet 2014}

BENOIT M., LAFON F., GOASGUEN G. (2008). Constitution et exploitation d'une base de données d'états de mer le long des côtes françaises par simulation numérique sur 23 ans. Base ANEMOC en Atlantique - Manche - Mer du Nord. European Journal of Env. and Civil Eng., Vol. 12/1-2, pp 35-50. http://dx.doi.org/10.1080/19648189.2008.9692994

BIDLOT J.-R., JANSSEN P. A. E. M., ABDALLA S. (2007). A revised formulation of ocean wave dissipation and its model impact. ECMWF Tech. Rep. Memo 509. Reading (UK).

BOUDIERE E., MAISONDIEU C., ARDHUIN F., ACCENSI M., PINEAUGUILLOU L., LEPESQUEUR J. (2013). A suitable metocean hindcast database for the design of marine energy converters. International Journal of Marine Energy, Vol. 3-4, pp 40-52. http://dx.doi.org/10.1016/j.ijome.2013.11.010

CHARLES E., IDIER D., THIEBOT J., LE COZANNET G., PEDREROS R., ARDHUIN F., PLANTON S. (2012). Present wave climate in the Bay of Biscay: spatiotemporal variability and trends from 1958-2001. Journal of Climate, Vol. 25, pp 2020-2039. http://dx.doi.org/10.1175/JCLI-D-11-00086.1

DODET G., BERTIN X., TABORDA R. (2010). Wave climate variability in the NorthEast Atlantic Ocean over the last six decades. Ocean Modelling, Vol. 31, pp 120-131. http://dx.doi.org/10.1016/j.ocemod.2009.10.010

HASSELMANN K. et al. (1973). Measurements of wind-wave growth and swell decay during the Joint North Sea Wave Project (JONSWAP). Deutschen Hydrographischen Zeitschrift.

HASSELMANN S., HASSELMANN K. (1985). Computations and parameterizations of the nonlinear energy transfer in gravity-wave spectrum. Part I: a new method for efficient computations of the exact nonlinear transfer integral. Journal of Physical Oceanography, Vol. 15, pp 1369-1377. http://dx.doi.org/10.1175/15200485(1985)015<1369:CAPOTN>2.0.CO;2

GUEDES SOARES C., CARRETERO-ALBIACH J.C., WEISSE R., ALVAREZFANJUL E. (2002) A 40 years hindcast of wind, sea level and waves in European waters. Proceedings of the 21st International Conference on Offshore Mechanics and Arctic Engineering, Oslo, (Norway), pp 669-675. http://dx.doi.org/10.1115/OMAE2002-28604 JANSSEN P.A.E.M. (1989). Wave induced stress and the drag of air flow sea waves. Journal of Physical Oceanography, Vol. 19, pp 745-754. http://dx.doi.org/10.1175/15200485(1989)019<0745:WISATD>2.0.CO;2

JANSSEN P.A.E.M. (1991). Quasi-linear theory of wind-wave generation applied to wave forecasting. Journal of Physical Oceanography, Vol. 21, pp 1631-1642. http://dx.doi.org/10.1175/1520-0485(1991)021<1631:QLTOWW>2.0.CO;2

KALNAY E. et al. (1996). The NCEP/NCAR 40-year Reanalysis Project. Bulletin of the American Meteorological Society, Vol. 77, pp 437-471. http://dx.doi.org/10.1175/15200477(1996)077<0437:TNYRP>2.0.CO;2 
KOMEN G.J., HASSELMANN S., HASSELMANN K. (1984). On the existence of a fully developed wind-sea spectrum. Journal of Physical Oceanography, Vol. 14, pp 1271-1285. http://dx.doi.org/10.1175/1520-0485(1984)014<1271:OTEOAF>2.0.CO;2

MORELLATO D., BENOIT M. (2009). Construction d'une base de données d'états de mer le long des côtes françaises méditerranéennes par simulations rétrospectives couvrant la période 1979-2008. Conférence CM2, Hammamet, Tunisie. http://dx.doi.org/10.5150/cmcm.2009.037-4

QUEFFEULOU P., CROIZE-FILLON D. (2009). La mesure satellite de hauteur de vague par altimètre. Etat des lieux, application à la climatologie et à la modélisation des états de mer. Proc. AMA 2009. Les Ateliers de Modélisation de l'Atmosphère, Toulouse.

REGUERO B.G., MENENDEZ M., MÉNDEZ F.J., MINGUEZ R., LOSADA I.J. (2012). A Global Ocean Wave (GOW) calibrated reanalysis from 1948 onwards. Coastal Engineering, Vol. 65, pp 38-55. http://dx.doi.org/10.1016/j.coastaleng.2012.03.003

SAHA S. et al. (2010). The NCEP Climate Forecast System Reanalysis. Bulletin of the American Meteorological Society, Vol. 91, pp 1015-1057. http://dx.doi.org/10.1175/2010BAMS3001.1

THORNTON E.B., GUZA R.T. (1983). Transformation of wave height distribution. Journal of Geophysical Research, Vol. 88, pp 5925-5938. http://dx.doi.org/10.1029/JC088iC10p05925

UPPALA S. M. et al. (2005). The ERA-40 re-analysis. Quarterly Journal of the Royal Meteorological Society, Vol. 18, pp 277-293. http://dx.doi.org/10.1256/qi.04.176 